\title{
THE EFFECT OF PREOPERATIVE ANXIETY WITH DEPTH OF ANESTHESIA DURING OOCYTE RETRIEVAL ON IVF SUCCESS
}

Sevtap Hekimoglu Sahin ${ }^{1}$, Elif Copuroglu ${ }^{1}$, Koray Elter ${ }^{2}$, Ozge Yaman ${ }^{2}$, Necdet Sut $^{3}$, Ece Yamak $^{1}$, Beyhan Karamanlioglu ${ }^{1}$

${ }^{1}$ Department of Anesthesiology and Reanimation, Trakya University Medical Faculty, Edirne, Turkey ${ }^{2}$ Department of Obstetrics and Gynecology, Trakya University Medical Faculty, Edirne, Turkey ${ }^{3}$ Department of Bioistatistics, Trakya University Medical Faculty, Edirne, Turkey

Purpose: Successful IVF is the goal of infertil women. This status is usually accompanied psychological and behavioral changes and can result in preoperative anxiety in women. Preoperative anxiety can negative impact on depth of anesthesia and IVF success. Furthermore, in the absence of premedication for oocyte retrieval, severe degrees of anxiety may occur. Anxiety effects the total consumption of analgesic and anesthetic drugs intraoperatively and has a negative impact on recovery from anesthesia. In addition, the negative effect of anesthetic agents used on fertilization and embryo quality during in vitro fertilization (IVF) has been discussed. However, the impact of any of them on fertilization and embryo quality has not been clearly determined to date. Studies have reported different results regarding the negative effects of anesthetic agents on embryo development and fertilization. Monitoring brain function gives real-time information to the depth of anesthesia of a patient. Patient State Index (PSI) monitoring has been used widely to determine anesthetic depth. It has been known that the PSI monitoring is helpful in reducing anxiety, prevention awareness, and harmful effects of an overdose of anesthesia.

The aim of this study was to evaluate the effect of preoperative anxiety with depth of anesthesia on IVF success.

Methods One hundred thirty-one patients between 25 and 43 years of age who had oocyte retrieval were enrolled in this study. The patients were divided according to Beck Anxiety Inventory (BAI): patients without anxiety were enrolled in the low-anxious group (group L) and patients with anxiety were included in the high-anxious patient group (group $\mathrm{H}$ ). Anesthesia was maintained with propofol infusion by ventilation with face mask, that was titrated to keep PSI value between 40 and 60. Demographic characteristics, Duration of surgery, fertilization rate and good quality embryo (GQE) rate, and adverse effects were 
recorded. Total propofol and fentanyl consumption was assessed at intraoperatively and the visual analogue scale (VAS) was recorded at $0,1,2$, and $4 \mathrm{~h}$ post-operatively.

The Modified Aldrete Score (MAS) was used to assess patients' recovery from anesthesia. When MAS was equal to or more than 9, patients were transferred to the clinic. The time for MAS to reach 9 was recorded.

The good quality embryo (GQE) rate and fertilization rate were evaluated. The fertilization rate was described as the number of fertilized oocytes (=2 pronuclei, $\mathrm{PN})$ divided by the number of retrieved oocytes. GQE rate was described as the number of GQEs divided by the number of 2 PN zygotes. A GQE was defined as having one 4 to 6 cells on the 2 nd day, 6-10 cells on 3rd day with $<20 \%$ fragmentation and no multinucleation, and finally a tightly packed inner cell mass and trophectoderm cells in a cohesive layer on the 5 th day. ${ }^{8}$ The fertilization rate and GQE rate were calculated per patient. The pregnancy rate was the secondary outcome.

Results: The groups were similar with respect to age, body mass index, duration of surgery and patient characteristics. The fertilization rate and GQE rate were not significant between the group L and group $\mathrm{H}(\mathrm{p}=0,848, \mathrm{p}=0,349)$, respectively. Mean number of embryos transferred, day of embryo transfer, and pregnancy rate were similar between the groups. Total propofol consumption was significantly higher in group $H$ than in groups $L(p=0,006)$. The HR at preoperative and postoperatively and SAP at preoperatve and DAP at postoperative were significantly increased in groups $\mathrm{H}$ than in group $\mathrm{L}(p=0,002, p<0,046, p<0,040$, and $p<0,025$, respectively). The time for MAS to reach 9 was significantly increased in group $\mathrm{H}$ than in group $\mathrm{L}(\mathrm{P}<0,001)$. The effect of variables that were found significantly in the univariate analysis (Propofol, $\mathrm{HR}_{\text {preop }}, \mathrm{HR}_{\text {postop }}, \mathrm{SBP}_{\text {preop }}, \mathrm{DBP}_{\text {postop }}$, and MAS reach of time 9) on BAI score. PSI scores at peroperative were similar in groups L and H. Total fentanyl consumption and VAS scores were not significant between groups.

Conclusion: Using PSI monitoring, we think that the increase total amount of propofol consumed due to increase in high anxiety will not have negative effects on fertilization and embryo quality or pregnancy rate. Therefore, in the high-anxious patient group, the increased need for intraoperative the amount of propofol can be accurately provided by PSI monitoring. 